\title{
molecules
}

ISSN 1420-3049

www.mdpi.com/journal/molecules

Article

\section{Inhibition of 11 $\beta$-HSD1 by Tetracyclic Triterpenoids from Euphorbia kansui}

\author{
Jie Guo ${ }^{1,2,3}$, Li-Yan Zhou ${ }^{1,2}$, Hong-Ping He ${ }^{3}$, Ying Leng ${ }^{4}$, Zhen Yang ${ }^{1,2}$ and Xiao-Jiang Hao ${ }^{3, *}$ \\ 1 Laboratory of Chemical Genomics, School of Chemical Biology and Biotechnology, \\ Peking University Shenzhen Graduate School, Shenzhen 518055, Guangdong, China; \\ E-Mails: guojie@pkusz.edu.cn (J.G.); zhouly@pkusz.edu.cn (L.-Y.Z.); zyang@pku.edu.cn (Z.Y.)
}

2 Key Laboratory of Bioorganic Chemistry and Molecular Engineering, Ministry of Education and Beijing National Laboratory for Molecular Science, College of Chemistry, Peking University, Beijing 100871, China

3 State Key Laboratory of Phytochemistry and Plant Resources in West China, Kunming Institute of Botany, Chinese Academy of Sciences, Kunming 650204, Yunnan, China; E-Mail: hehongping@mail.kib.ac.cn

4 Shanghai Institute of Materia Medica, Chinese Academy of Sciences, Shanghai 201203, China; E-Mail: yleng@mail.shcnc.ac.cn

* Author to whom correspondence should be addressed; E-Mail: haoxj@mail.kib.ac.cn; Tel.: +86-871-522-3263; Fax: +86-871-521-9684.

Received: 6 August 2012; in revised form: 13 September 2012 / Accepted: 18 September 2012 / Published: 9 October 2012

\begin{abstract}
The roots of Euphorbia kansui are considered an important traditional folk medicine. In this study the ethanol extracts of E. kansui were investigated. A new tetracyclic triterpenoid, euphane-33,20-dihydroxy-24-ene, in addition to five known triterpenoids with euphane skeletons were isolated. Their structures were elucidated on the basis of physical and spectral techniques (1D-, 2D-NMR and MS, respectively). Furthermore, these compounds 1-6 exhibited strong inhibitory activity against human $11 \beta$-hydroxysteroid dehydrogenase type 1 (11 $\beta$-HSD1), with $\mathrm{IC}_{50}$ values of $34.86 \mathrm{nM}$, $1.115 \mu \mathrm{M}, 16.08 \mathrm{nM}, 2.815 \mathrm{nM}, 26.47 \mathrm{nM}, 15.99 \mathrm{nM}$, and $41.86 \mathrm{nM}$, respectively. The docking results show that the ring part of compounds can insert into the hydrophobic core of h11 $\beta$-HSD1 and the alkane chain orientates toward the outside. The results presented herein provide a scientific explanation for the usage of the E. kansui in clinical treatment of diabetes.
\end{abstract}




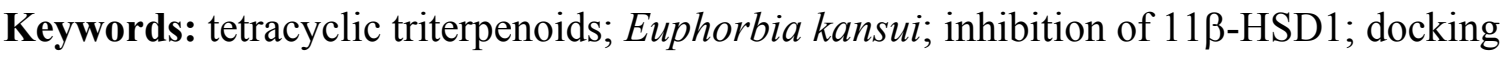

\section{Introduction}

Type 2 diabetes mellitus is a complex endocrine and metabolic disorder. The interaction between several genetic and environmental factors results in a heterogeneous and progressive disorder with variable degrees of insulin resistance and pancreatic $\beta$-cell dysfunction [1]. In China it is proving to be a major public health problem, especially in the urban areas. The increasing prevalence, variable pathogenesis natural history, and complications of type 2 diabetes emphasize the urgent need for new treatment strategies [2]. Herbal supplements for diabetes should be a part of a holistic approach to treatment that addresses proper nutrition, a good exercise program, and continued monitoring of blood glucose levels.

Plants of the Euphorbia genus produce structurally unique and diversified diterpenoids and triterpenoids, which have attracted great interest from the biogenetic, synthetic, biological and toxicological points of view [3-7]. Certain types of triterpenoids isolated from plants of the genus Euphorbia, such as euphanes and tirucallanes, may be the ultimate biogenetic precursors of limonoids which have recently attracted attention because compounds belonging to this group have exhibited a range of biological activities like insecticidal, insect antifeedant and growth regulating activity on insects as well as antibacterial, antifungal, antimalarial, anticancer, antiviral and a number of other pharmacological activities in humans [8]. Therefore, the triterpenoids and its derivatives produced by genus Euphorbia have a lot of future in studies of chemical components and biological activities.

Euphorbia kansui Lour. (Euphorbiaceae) is a vivacious herb distributed in the central and western parts of China. The dried roots of Euphorbia kansui have been used as an herbal remedy for edema, ascites, and cancer in mainland China [9]. Previous phytochemical investigations on this species yielded a number of ingenol diterpenoid esters and jatrophane diterpenoids [10,11]. In the course of our search for bioactive natural products from the roots of E. kansui, six tetracyclic triterpenoids 1-6 (Figure 1) were isolated from the EtOH extracts of this plant. Herein we describe the isolation and characterization of the new triterpenoid, along with five known triterpenoids, and the evaluation of inhibitory activity against human and mouse $11 \beta$-HSD1.

\section{Results and Discussion}

\subsection{Identification of Compounds}

Compound 1 was obtained as a colorless powder. Its molecular formula was determined to be $\mathrm{C}_{31} \mathrm{H}_{54} \mathrm{O}_{2}$ by HR-TOF-MS $\left(\mathrm{m} / z[\mathrm{M}+\mathrm{Na}]^{+} 481.4173\right.$, calcd. 481.4124). The IR absorption bands indicated the presence of double bond $\left(1639 \mathrm{~cm}^{-1}\right)$ and hydroxyl $\left(3439 \mathrm{~cm}^{-1}\right)$ groups. The 1D NMR spectra exhibited resonances for six quaternary, eleven methylene, six methine, and eight methyl carbons, which were assigned to a terminal double bond, one oxygenated quaternary, one oxygenated methine, two secondary methyls, six tertiary methyls, and two hydroxyl groups. As the molecular 
formula indicated the presence of five units of unsaturation, the compound must therefore be tetracarbocyclic since there is only one terminal double group.

Figure 1. Structures of the triterpenoids 1-6 isolated from Euphorbia kansui.
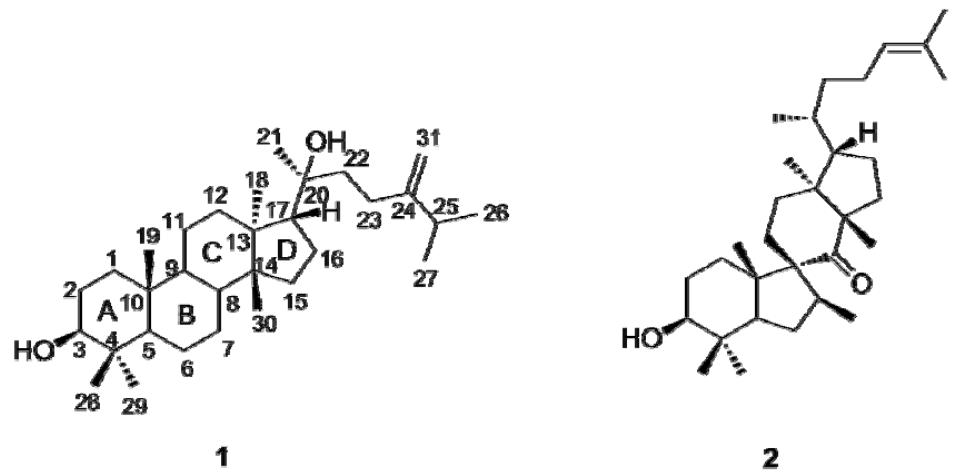

2

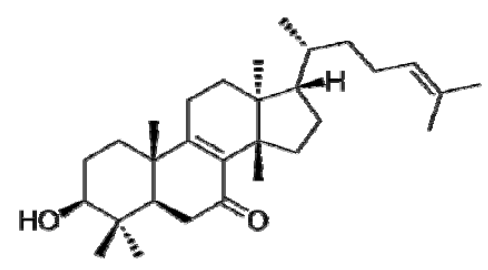

4

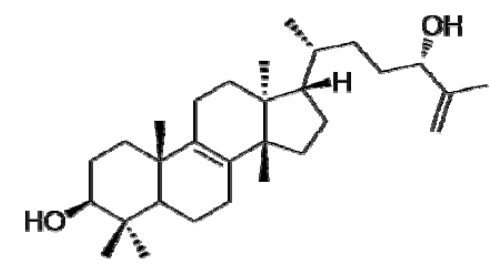

5

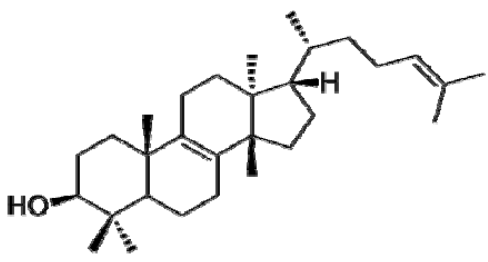

3

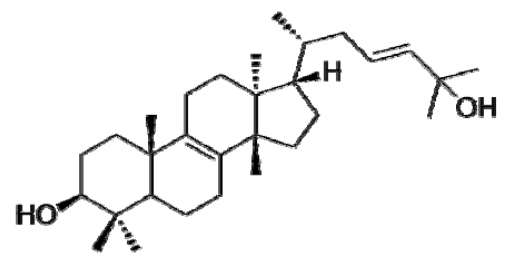

6

Comparison of the ${ }^{1} \mathrm{H}$ - and ${ }^{13} \mathrm{C}-\mathrm{NMR}$ data of $\mathbf{1}$ with those of euphorbol [12], a tirucallane-type triterpenoid isolated from the same plant, revealed that both compounds were characterized by similar chemical shifts, suggesting a common structural motif they shared, except for the signals due to the olefinic group and the side chain part. Two hydroxyls, as required by its molecular formula and IR spectra, were located at positions $\mathrm{C}-3$ and $\mathrm{C}-20$ based on their chemical shifts and HMBC correlations from $\mathrm{H}-3$ to Me-28,29, and Me-21 to C-20. The detailed analysis of 1 using ${ }^{1} \mathrm{H}-{ }^{1} \mathrm{H}$ COSY and HMQC techniques disclosed three partial structural units, between $\mathrm{H}-3$ and $\mathrm{H}-2$; between $\mathrm{H}-2$ and $\mathrm{H}-1$; between $\mathrm{H}-5$ and H-6; between $\mathrm{H}-6$ and $\mathrm{H}-7$; between $\mathrm{H}-7$ and $\mathrm{H}-8$; between $\mathrm{H}-8$ and $\mathrm{H}-9$; between H-9 and H-11; between H-11 and H-12, and between H-15 and H-16, and between H-16 and H-17. This was also supported by analysis of the HMBC spectrum, which showed two- and three-bond correlations between $\mathrm{H}-1$ and C-2; between $\mathrm{H}-2$ and C-3; between $\mathrm{H}-5$ and C-6; between $\mathrm{H}-6$ and C-7; between $\mathrm{H}-7$ and $\mathrm{C}-8$; between $\mathrm{H}-8$ and $\mathrm{C}-9$; between $\mathrm{H}-9$ and $\mathrm{C}-11$; between $\mathrm{H}-11$ and $\mathrm{C}-12$; between $\mathrm{H}-15$ and C-17; and between $\mathrm{H}-16$ and C-17.

Furthermore, the HMBC correlations of the five individual tertiary methyl signals on rings A-D [between Me-28 $\left(\delta_{\mathrm{H}} 0.96\right)$ and C-29, C-4, C-3, and C-5; between Me-29 ( $\left.\delta_{\mathrm{H}} 0.77\right)$ and C-28, C-4, C-3, and C-5; between Me-19 $\left(\delta_{\mathrm{H}} 0.84\right)$ and C-1, C-5, C-9, and C-10; between Me-30 $\left(\delta_{\mathrm{H}} 0.88\right)$ and C-8, C-13, $\mathrm{C}-14$, and $\mathrm{C}-15$; between Me-18 ( $\left.\delta_{\mathrm{H}} 0.96\right)$ and $\mathrm{C}-12, \mathrm{C}-13, \mathrm{C}-14$, and C-17] firmly established the linkages of these partial structural units (substructure a). The side chain (substructure $\mathrm{b}$ ) was assembled by ${ }^{1} \mathrm{H}-{ }^{1} \mathrm{H}$ COSY correlations between $\mathrm{H}-25 / \mathrm{Me}-26,27$ and $\mathrm{H}-22 / \mathrm{H}-23$ as well as $\mathrm{HMBC}$ correlations of H-31/C-25, H-31/C-23, Me-26,27/C-24, Me-21/C-22, H-22/C-20 and Me-21/C-20. The two substructures should join at $\mathrm{C}-17$ confirmed by the HMBC cross peaks between Me-21 and C-17 and between H-16 and C-20. Therefore, a planar structure of $\mathbf{1}$ was derived, as shown in Figure 2. 
Figure 2. Selected two-dimensional NMR correlations of compound $\mathbf{1 .}$

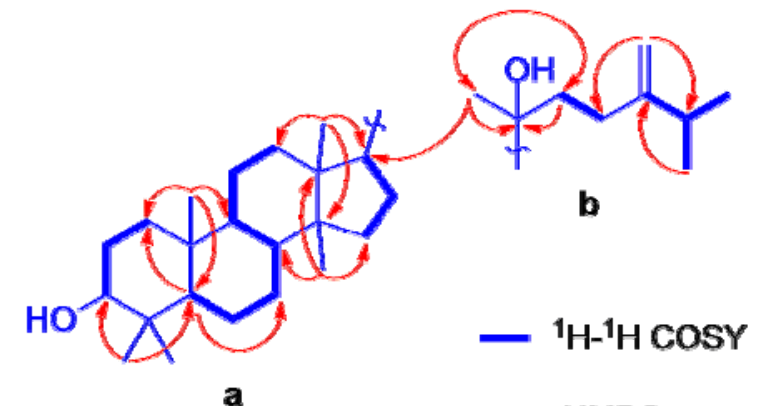

HMBC

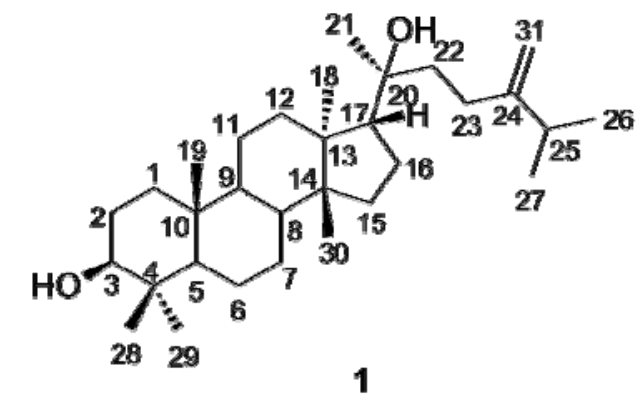

1

The relative configuration of $\mathbf{1}$ was determined by ROESY experiments (Figure 3). The large coupling constant $\left(J_{2,3}=10.8 \mathrm{~Hz}\right)$ of $\mathrm{H}-3$ indicated that the hydroxyl group was oriented equatorially $(\beta)$ at $\mathrm{C}-3$ [13]. The relative configurations of the methyl groups and other protons in the rings A-D were ascertained on the basis of the ROESY correlations. The significant ROESY correlations of $\mathrm{H}-3 / \mathrm{H}-5, \mathrm{H}-3 / \mathrm{Me}-28, \mathrm{H}-5 / \mathrm{H}-9$, and Me-18/H-9 indicated that $\mathrm{H}-3, \mathrm{H}-5, \mathrm{Me}-28, \mathrm{H}-9$, and Me-18 were cofacial, adopting an $\alpha$-orientation. The ROESY cross-peaks of Me-19/H-1 $\beta$, Me-19/H-12 $\beta$, Me-30/H$12 \beta$, and Me-30/H-17 indicated that Me-19, Me-30 and H-17 was $\beta$-oriented. Furthermore, ROESY correlations between Me-21 and $\mathrm{CH}_{2}-16$ and the absence of NOE between Me-18 and Me-21 were consistent with those of euphane-type triterpenoids [13,14]. And the positive optical rotation of 1 $\left(+13.4^{\circ}\right)$ also indicated that $\mathbf{1}$ belonged to the euphane rather than the tirucallane series [15-17]. Thus, compound 3 was elucidated to be euphane-3 $\beta, 20$-dihydroxy-24-ene.

Figure 3. Key ROESY correlations of compound $\mathbf{1 .}$

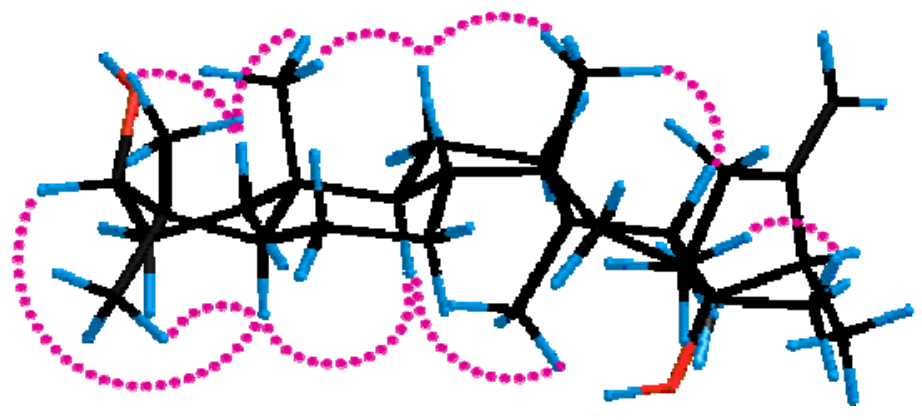

\subsection{Inhibition of $11 \beta-H S D 1$}

The oxidoreductase $11 \beta$-hydroxysteroids dehydrogenase type 1 (11ß-HSD1) mainly catalyzes the intracellular regeneration of active GCs (cortisol, corticosterone) from inert inactive 11-keto forms (cortisone) in liver, adipose tissue and brain, amplifying local GC action. Multiple lines of evidence have indicated that $11 \beta$-HSD1-mediated intracellular cortisal production may have a pathogenic role in type 2 diabetes and its co-morbidities. $11 \beta$-HSD1 becomes a novel target for anti-type 2 diabetes drug development, and inhibition of $11 \beta$-HSD1 offers a potential therapy to attenuate the type 2 diabetes [18].

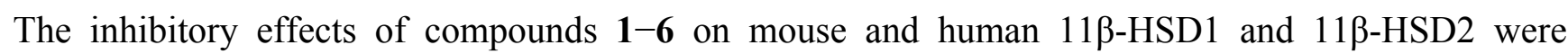
evaluated (Table 1). All assays were carried out in duplicate with glycyrrhizinic acid and carbenoxolone as positive controls. All the tested compounds 1-6 have a significant inhibition of both mouse and human 
$11 \beta$-HSD1, among them compound 4 shows the strongest inhibitory effect on mouse and human 11ß-HSD1 inhibition with $\mathrm{IC}_{50}$ of 13.36 and $2.815 \mathrm{nM}$, respectively. Compounds $\mathbf{2}-\mathbf{5}$ have $\mathrm{IC}_{50}>1 \mathrm{mM}$ against mouse $11 \beta$-HSD2; however, only compound $\mathbf{1}$ has a good selectivity against human $11 \beta$-HSD2, the $\mathrm{IC}_{50}$ was $8.179 \mu \mathrm{M}$ and selectivity between HSD2/HSD1 was 234.6 times, the remaining compounds HSD2/HSD1 selectivity are less than 100 times. In Traditional Chinese Medicine, processed E. kansui has been used as a herbal remedy for diabetes [19]. The results presented herein provide a scientific explanation for the usage of the E. kansui in clinical treatment.

Table 1. Inhibition of $11 \beta$-HSD1.

\begin{tabular}{lllllll}
\hline Compounds & $\begin{array}{l}\text { Mouse } \\
\mathbf{1 1} \boldsymbol{\beta}-\mathbf{H S D 1} \\
\left(\mathbf{I C}_{\mathbf{5 0}}\right)\end{array}$ & $\begin{array}{l}\text { Mouse } \\
\mathbf{1 1 \beta - H S D 2} \\
\left(\mathbf{I C}_{\mathbf{5 0}}\right)\end{array}$ & $\begin{array}{l}\text { Mouse } \\
\text { HSD2/HSD1 }\end{array}$ & $\begin{array}{l}\text { Human } \\
\mathbf{1 1} \boldsymbol{\beta}-\mathrm{HSD1} \\
\left(\mathbf{I C}_{\mathbf{5 0}}\right)\end{array}$ & $\begin{array}{l}\text { Human } \\
\mathbf{1 1} \boldsymbol{\beta}-\mathbf{H S D 2} \\
\left(\mathbf{I C}_{\mathbf{5 0}}\right)\end{array}$ & $\begin{array}{l}\text { Human } \\
\text { HSD2/HSD1 }\end{array}$ \\
\hline Compound 1 & $78.44 \mathrm{nM}$ & $>1 \mathrm{mM}$ & $>12748$ & $34.86 \mathrm{nM}$ & $8.179 \mu \mathrm{M}$ & 234.6 \\
Compound 2 & $1.077 \mu \mathrm{M}$ & $>1 \mathrm{mM}$ & $>928$ & $1.115 \mu \mathrm{M}$ & $2.626 \mu \mathrm{M}$ & 2.35 \\
Compound 3 & $80.52 \mathrm{nM}$ & $>1 \mathrm{mM}$ & $>12419$ & $16.08 \mathrm{nM}$ & $0.3952 \mu \mathrm{M}$ & 24.6 \\
Compound 4 & $13.36 \mathrm{nM}$ & $>1 \mathrm{mM}$ & $>74850$ & $2.815 \mathrm{nM}$ & $0.107 \mu \mathrm{M}$ & 38 \\
Compound 5 & $49.46 \mathrm{nM}$ & $>1 \mathrm{mM}$ & $>20218$ & $26.47 \mathrm{nM}$ & $1.687 \mu \mathrm{M}$ & 63.7 \\
Compound 6 & $294.7 \mathrm{nM}$ & $>1 \mathrm{mM}$ & $>3393$ & $15.99 \mathrm{nM}$ & $0.6664 \mu \mathrm{M}$ & 41.7 \\
\hline
\end{tabular}

\subsection{In Silico Study of the Activities of Compounds}

In order to investigate the activity difference against h11 $\beta$-HSD1 of these compounds, we predicted their binding mode using molecular docking. The docking results show that the binding modes of compound 1, 3,5 and $\mathbf{6}$ are similar (Figure 4). The ring part of compounds inserts into a hydrophobic core composed of Leu126, Leu171, Tyr177, Val180, Tyr183, Leu217, Ala223, Ala226, Val227, Val231, Met233 and the cofactor NADP. The alkane chain orientates towards the outside. The C-24 hydroxyl of compound 5 can form hydrogen bonds with the side chain of Asn123. The C-21 hydroxyl of compound $\mathbf{6}$ can form hydrogen bonds with the backbone carbonyl group of Thr124.

Figure 4. The binding mode of compounds 1,3,5, and 6. Purple: compound 1; Cyan: compound 3; Yellow: compound 5; Orange: compound 6.

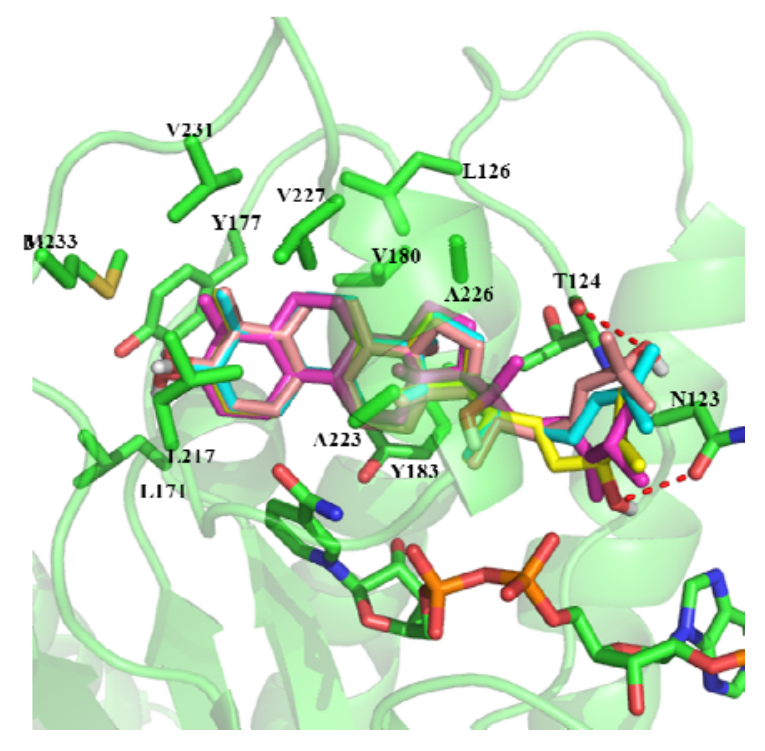


Compound $\mathbf{2}$ is a rearranged euphane triterpenoid containing a contracted five-membered ring B. Although the ring part of compound 2 can also insert into the hydrophobic core, the C-15 position has a steric clash with the residue Ala223. The ring part of compound $\mathbf{4}$ also can insert into the core composed of hydrophobic residues. The C-7 carbonyl of compound 4 can form hydrogen bonds with the side chain of Tyr183. Kelly et al. [20] have shown that hydrogen bonds can be stronger by up to $1.2 \mathrm{kcal} / \mathrm{mol}$ when they are sequestered in hydrophobic surroundings than when they are solvent exposed. This may be the reason that the activity of compound 4 is a little higher than that of the other compounds (Figure 5).

Figure 5. The binding mode of compound 2 and 4. Cyan: compound 3; Orange: compound 2; Yellow: compound 4.
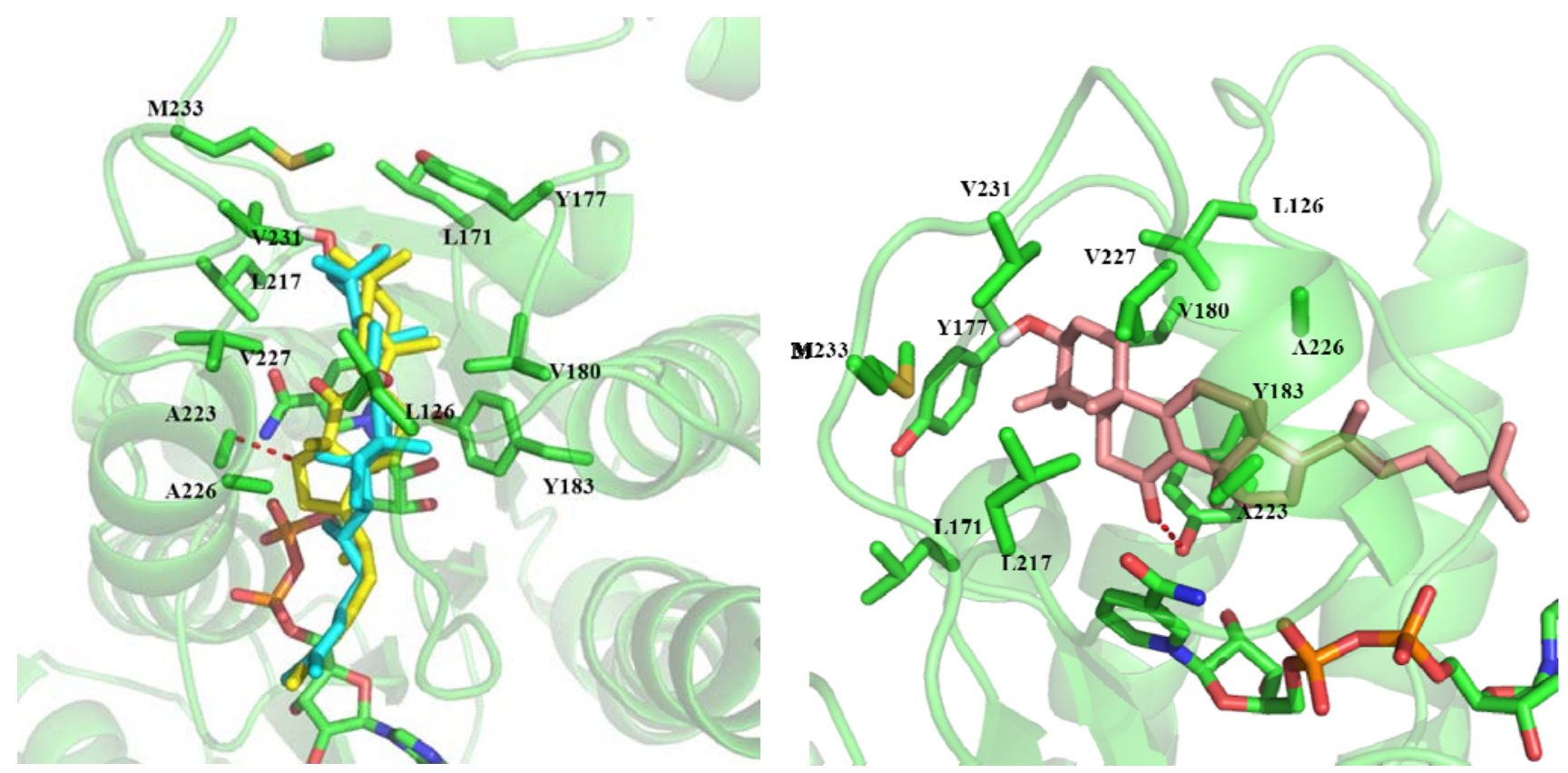

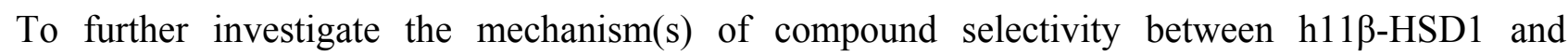

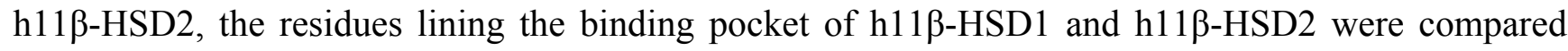
(Figure 6). It can be found that most of the binding pocket residues are similar except three residues

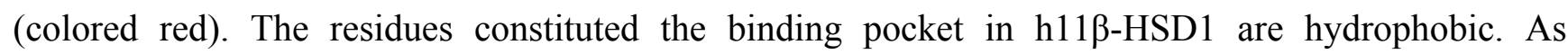

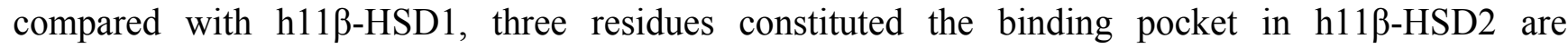
hydrophilic (Glu217, Glu226 and Lys227, colored red). These hydrophilic residues may affect that the

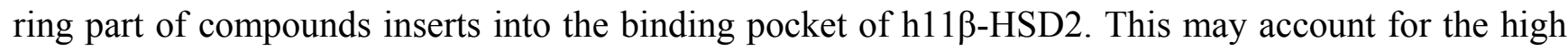
h11 $\beta$-HSD1 selectivity of these compounds. 
Figure 6. The sequence alignments of hHSD1 and hHSD2, the residues formed binding pocket were colored yellow.

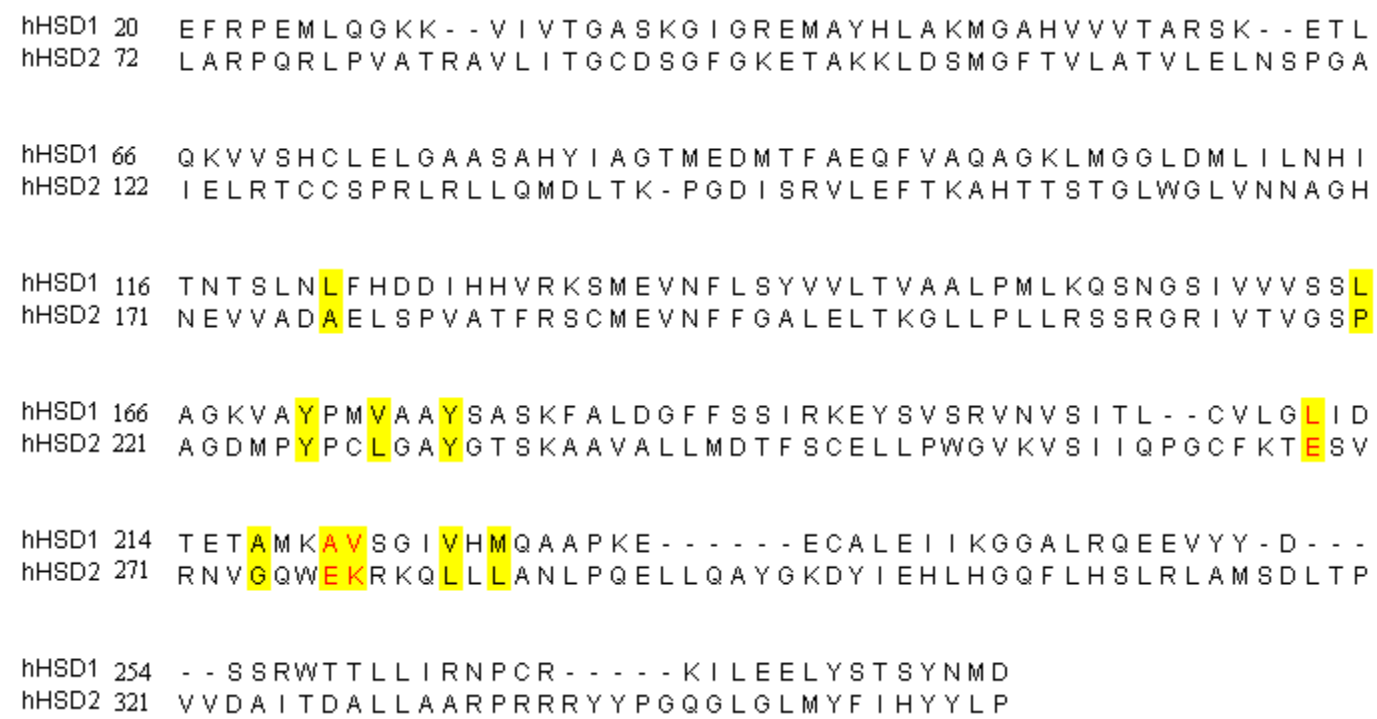

\section{Experimental}

\subsection{General}

Optical rotation was measured on a Perkin-Elmer model 241 polarimeter. The IR spectrum was measured in a Bio-Rad FTS-135 spectrometer with $\mathrm{KBr}$ pellets. FAB, EI and high-resolution mass spectra were recorded using a Finnigan MAT 90 instrument and VG Autospec-3000 spectrometer respectively. ${ }^{1} \mathrm{H}$ - and ${ }^{13} \mathrm{C}-\mathrm{NMR}$ spectra were measured on a Bruker AM-400 spectrometer, while 2D NMR spectra were recorded on a Bruker DRX-500 instrument. Chemical shifts were reported using residual $\mathrm{CDCl}_{3}\left(\delta_{\mathrm{H}} 7.26\right.$ and $\left.\delta_{\mathrm{C}} 77.0\right)$ as internal standard. Column chromatography was performed on silica gel H (10-40 $\mu \mathrm{m}$; Qingdao Marine Chemical Inc., Qiangdao, China), C18 silica gel (20-45 $\mu \mathrm{m}$; Chromatorex, Tokyo, Japan), Precoated silica gel GF254 and HF254 plates (Qingdao Haiyang Chemical Plant, Qingdao, China) were used for TLC.

\subsection{Plant Material}

The roots of Euphorbia kansui Lour. were collected in Kuitun of Gansu Province, China, in December 2007, and identified by Xun Gong, Kunming Institute of Botany, Chinese Academy Sciences, Kunming, Yunnan, China.

\subsection{Extraction and Isolation}

The dried and powdered roots of E. kansui $(20 \mathrm{~kg}$ ) were extracted with $95 \% \mathrm{EtOH}(40 \mathrm{~L}, 3$ times, $\left.60{ }^{\circ} \mathrm{C}\right)$. Removal of the solvent gave a crude residue $(760 \mathrm{~g})$, which was partitioned between petroleum ether $(280 \mathrm{~g})$, EtOAc $(56 \mathrm{~g})$, and $\mathrm{H}_{2} \mathrm{O}(420 \mathrm{~g})$. The petroleum ether extracts was applied to a silica gel column (200-300 mesh), eluting with gradient mixtures of petroleum ether-acetone (from 1:0 to 0:1) to give seven major fractions (Fr1-Fr7). Fr1 (80 g) was chromatographed on a silica gel (200-300 mesh) column eluted with petroleum-ether/EtOAc, (50:1) to afford 3 (979 mg). Fr5 (50 g) was subjected to 
amino silica gel and silica gel CC (300-400 mesh), eluting with petroleum ether/EtOAc (from 10:1 to $3: 1)$ to afford four major subfractions, Fr5a-Fr5d. Fr5b $(2.7 \mathrm{~g})$ was further purified by silica gel CC (silica gel H, petroleum ether/acetone, 18:1) and Sephadex LH-20 column (petroleum ether/ $\left.\mathrm{CHCl}_{3} / \mathrm{MeOH}, 2: 1: 1\right)$ to obtain 2 (18 mg) and $\mathbf{1}(42 \mathrm{mg})$. Fr6 (39 g) was applied to a MPLC, eluted with $\mathrm{CH}_{3} \mathrm{OH} / \mathrm{H}_{2} \mathrm{O}$ (3:5 to 1:0) to afford three major subfractions, Fr6a-Fr6c. Fr6a (2.2 g) was further purified by silica gel CC (silica gel H, petroleum ether/EtOAc, 20:1) and Sephadex LH-20 column (petroleum ether/ $\left.\mathrm{CHCl}_{3} / \mathrm{MeOH}, 2: 1: 1\right)$ to obtain $4(20 \mathrm{mg})$. The EtOAc $(56 \mathrm{~g})$ phase was subjected to column chromatogratography on RP-18 silica gel eluted with $\mathrm{MeOH} / \mathrm{H}_{2} \mathrm{O}$ (6:4 to pure $\mathrm{MeOH}$ ), to furnish five fractions (EFr1-EFr5). The fourth fraction $\left(\mathrm{MeOH} / \mathrm{H}_{2} \mathrm{O}\right.$ 9:1, $3 \mathrm{~g}$ ) was rechromatographed on a Sephadex LH-20 column $(\mathrm{MeOH})$ and further purified by silica gel CC (Silica gel H, petroleum ether/acetone, 12:1) to yield compounds 5 (12 mg) and 6 (5 mg).

Euphane-3 $\beta, 20$-dihydroxy-24-ene (1), Formula: $\mathrm{C}_{31} \mathrm{H}_{54} \mathrm{O}_{2}$; colorless oil; $[\alpha]_{\mathrm{D}}{ }^{20}+13.4^{\circ}(\mathrm{c} 0.30, \mathrm{MeOH})$; IR $v_{\max }(\mathrm{KBr}) 3439,3086,2958,2871,1044 \mathrm{~cm}^{-1}$; EI-MS m/z 458 [M] ${ }^{+}$; HR-TOF-MS m/z 481.4173. ${ }^{1} \mathrm{H}-\mathrm{NMR}: 1.70(1 \mathrm{H}, \mathrm{m}, 1 \alpha-\mathrm{H}), 0.96(1 \mathrm{H}, \mathrm{m}, 1 \beta-\mathrm{H}), 1.62\left(2 \mathrm{H}, \mathrm{m}, 2-\mathrm{CH}_{2}\right), 3.21(1 \mathrm{H}, \mathrm{dd}, J=4.8,10.8 \mathrm{~Hz}$, 3-H), $0.72(1 \mathrm{H}, \mathrm{d}, J=11.6 \mathrm{~Hz}, 5-\mathrm{H}), 1.46\left(2 \mathrm{H}, \mathrm{m}, 6-\mathrm{CH}_{2}\right), 1.74(1 \mathrm{H}, \mathrm{m}, 7 \alpha-\mathrm{H}), 1.33(1 \mathrm{H}, \mathrm{m}, 7 \beta-\mathrm{H})$, $1.72(1 \mathrm{H}, \mathrm{m}, 8-\mathrm{H}), 1.31(1 \mathrm{H}, \mathrm{m}, 9-\mathrm{H}), 1.50(1 \mathrm{H}, \mathrm{m}, 11 \alpha-\mathrm{H}), 1.26(\mathrm{H}, \mathrm{m}, 11 \beta-\mathrm{H}), 1.26(1 \mathrm{H}, \mathrm{m}, 12 \alpha-\mathrm{H})$, $1.57(1 \mathrm{H}, \mathrm{m}, 12 \beta-\mathrm{H}), 1.47(1 \mathrm{H}, \mathrm{m}, 15 \alpha-\mathrm{H}), 1.07(1 \mathrm{H}, \mathrm{m}, 15 \beta-\mathrm{H}), 1.88(1 \mathrm{H}, \mathrm{m}, 16 \alpha-\mathrm{H}), 1.26(1 \mathrm{H}, \mathrm{m}$, $16 \beta-\mathrm{H}), 1.75(1 \mathrm{H}, \mathrm{m}, 17-\mathrm{H}), 0.96(3 \mathrm{H}, \mathrm{s}, 18-\mathrm{Me}), 0.84(3 \mathrm{H}, \mathrm{s}, 19-\mathrm{Me}), 1.13(3 \mathrm{H}, \mathrm{s}, 21-\mathrm{Me}), 1.57(1 \mathrm{H}$, $\left.\mathrm{m}, 22-\mathrm{CH}_{2}\right), 2.10\left(2 \mathrm{H}, \mathrm{m}, 23-\mathrm{CH}_{2}\right), 2.25(1 \mathrm{H}$, septet, 25-H), $1.06(6 \mathrm{H}, \mathrm{d}, J=6.0 \mathrm{~Hz}, 26-\mathrm{Me}$ and 27- Me), $0.97(3 \mathrm{H}, \mathrm{s}, 28-\mathrm{Me}), 0.77$ (3H, s, 29- Me), 0.88 (3H, s, 30- Me), 4.74 and $4.68\left(2 \mathrm{H}, \mathrm{s}, 31-\mathrm{CH}_{2}\right) .{ }^{13} \mathrm{C}-\mathrm{NMR}$ : 39.0 (C-1), 27.3 (C-2), 78.9 (C-3), 38.9 (C-4), 55.8 (C-5), 18.4 (C-6), 25.3 (C-7), 42.1 (C-8), 50.4 (C-9), 37.2 (C-10), 21.4 (C-11), 32.5 (C-12), 49.9 (C-13), 40.3 (C-14), 31.0 (C-15), 27.5 (C-16), 49.5 (C-17), 16.5 (C-18), 15.6 (C-19), 75.7 (C-20), 23.7 (C-21), 40.5 (C-22), 28.0 (C-23), 156.4 (C-24), 34.1 (C-25), 21.9 (C-26), 21.9 (C-27), 28.0 (C-28), 15.4 (C-29), 16.1 (C-30), 106.1 (C-31).

Kansuinone (2), Formula: $\mathrm{C}_{30} \mathrm{H}_{50} \mathrm{O}_{3}$; colorless oil; $[\alpha]_{\mathrm{D}}{ }^{16}+12.4^{\circ}$ (c $\left.0.19, \mathrm{MeOH}\right)$; UV (MeOH) $\lambda \max$ $202.2 \mathrm{~nm}$; IR (KBr) v $v_{\text {max }}: 3432,2963,2928,1675,1629,1460,1378,1022$ and $584 \mathrm{~cm}^{-1}$; EI-MS $\mathrm{m} / z$ $458[\mathrm{M}]^{+}$; the ${ }^{1} \mathrm{H}-\mathrm{NMR}$ and ${ }^{13} \mathrm{C}-\mathrm{NMR}$ data in accordance with the literature [21]. ${ }^{1} \mathrm{H}$ NMR: $2.10(1 \mathrm{H}$, $\mathrm{m}, 1 \alpha-\mathrm{H}), 1.20(1 \mathrm{H}, \mathrm{m}, 1 \beta-\mathrm{H}), 1.67(2 \mathrm{H}, \mathrm{m}, 2-\mathrm{H}), 3.49(1 \mathrm{H}, \mathrm{dd}, J=6.4,9.2 \mathrm{~Hz}, 3-\mathrm{H}), 2.68(1 \mathrm{H}, \mathrm{dd}$, $J=14.4,6.0 \mathrm{~Hz}, 5-\mathrm{H}), 2.16(1 \mathrm{H}, \mathrm{m}, 6 \alpha-\mathrm{H}), 1.43(1 \mathrm{H}, \mathrm{m}, 6 \beta-\mathrm{H}), 4.26(1 \mathrm{H}, \mathrm{t}, J=7.2 \mathrm{~Hz}, 7-\mathrm{H}), 2.13$ $(1 \mathrm{H}, \mathrm{m}, 11 \alpha-\mathrm{H}), 1.64(1 \mathrm{H}, \mathrm{m}, 11 \beta-\mathrm{H}), 1.88(1 \mathrm{H}, \mathrm{m}, 12 \alpha-\mathrm{H}), 1.79(1 \mathrm{H}, \mathrm{m}, 12 \beta-\mathrm{H}), 1.76(1 \mathrm{H}, \mathrm{m}, 15 \alpha-\mathrm{H})$, $1.30(1 \mathrm{H}, \mathrm{m}, 15 \beta-\mathrm{H}), 1.31(1 \mathrm{H}, \mathrm{m}, 16 \alpha-\mathrm{H}), 1.88(1 \mathrm{H}, \mathrm{m}, 16 \beta-\mathrm{H}), 1.62(1 \mathrm{H}, \mathrm{m}, 17-\mathrm{H}), 0.69(3 \mathrm{H}, \mathrm{s}, 18-\mathrm{H})$, $0.90(3 \mathrm{H}, \mathrm{s}, 19-\mathrm{H}), 1.46(1 \mathrm{H}, \mathrm{m}, 20-\mathrm{H}), 0.86(3 \mathrm{H}, \mathrm{d}, J=6.4 \mathrm{~Hz}, 21-\mathrm{H}), 1.55(1 \mathrm{H}, \mathrm{m}, 22 \alpha-\mathrm{H}), 1.13(1 \mathrm{H}$,

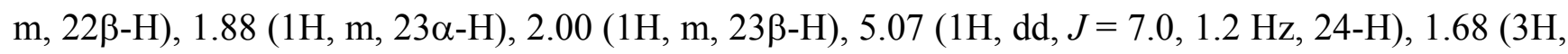
s, 26-H), $1.60(3 \mathrm{H}, \mathrm{s}, 27-\mathrm{H}), 0.99(3 \mathrm{H}, \mathrm{s}, 28-\mathrm{H}), 0.88(3 \mathrm{H}, \mathrm{s}, 29-\mathrm{H}), 1.16(3 \mathrm{H}, \mathrm{s}, 30-\mathrm{H}) .{ }^{13} \mathrm{C}-\mathrm{NMR}: 29.7$ (C-1), 28.2 (C-2), 79.3 (C-3), 37.8 (C-4), 47.8 (C-5), 34.7 (C-6), 76.4 (C-7), 219.3 (C-8), 61.6 (C-9), 49.0 (C-10), 24.2 (C-11), 31.5 (C-12), 45.9 (C-13), 61.8 (C-14), 29.7 (C-15), 26.8 (C-16), 49.6 (C-17), 16.8 (C-18), 17.6 (C-19), 35.2 (C-20), 18.6 (C-21), 35.3 (C-22), 24.6 (C-23), 124.8 (C-24), 131.5 (C-25), 25.7 (C-26), 17.7 (C-27), 29.7 (C-28), 16.6 (C-29), 22.4 (C-30). 
Euphol (3), Formula: $\mathrm{C}_{30} \mathrm{H}_{50} \mathrm{O}$; colorless needle; m.p. $165-167{ }^{\circ} \mathrm{C}$; $[\alpha]_{\mathrm{D}}{ }^{20}+32.0^{\circ}$ (c $\left.0.30, \mathrm{MeOH}\right)$; ESI-MS $m / z: 427[\mathrm{M}+\mathrm{H}]^{+}, 411,393,109,69$; the ${ }^{1} \mathrm{H}-\mathrm{NMR}$ and ${ }^{13} \mathrm{C}-\mathrm{NMR}$ data in accordance with the literature [22]. ${ }^{1} \mathrm{H}-\mathrm{NMR}: 0.75(3 \mathrm{H}, \mathrm{s}, 18-\mathrm{H}), 0.79(3 \mathrm{H}, \mathrm{s}, 29-\mathrm{H}), 0.84(3 \mathrm{H}, \mathrm{s}, 30-\mathrm{H}), 0.86(3 \mathrm{H}, \mathrm{d}$, $J=6.4 \mathrm{~Hz}, 2 \mathrm{l}-\mathrm{H}), 0.95$ (3H, s, 28-H), 1.00 (3H, s, 19-H), 1.60 (3H, s, 26-H), 1.66 (3H, s, 27-H), 3.21 $(1 \mathrm{H}, \mathrm{dd}, J=11.5,4.5 \mathrm{~Hz}, 3-\mathrm{H}), 5.08(1 \mathrm{H}, \mathrm{t}, J=7.0 \mathrm{~Hz}, 24-\mathrm{H}) .{ }^{13} \mathrm{C}-\mathrm{NMR}: 35.73$ (C-1), 24.28 (C-2), 79.06 (C-3), 38.96 (C-4), 50.61 (C-5), 18.35 (C-6), 27.89 (C-7), 134.58 (C-8), 134.58 (C 9), 36.29 (C-10), 21.09 (C-11), 28.22 (C-12), 44.65 (C-13), 49.93 (C-14), 30.93 (C-15), 31.16 (C-16), 50.56 (C-17), 15.82 (C-18), 19.16 (C-19), 36.44 (C-20), 18.69 (C-21), 35.64 (C-22), 25.01 (C-23), 125.34 (C-24), 131.00 (C-25), 17.59 (C-26), 25.64 (C-27), 26.61 (C-28), 28.01 (C-29), 15.40 (C-30).

Kansenone (4), Formula: $\mathrm{C}_{30} \mathrm{H}_{48} \mathrm{O}_{2}$; colorless oil; $[\alpha]_{\mathrm{D}}{ }^{20}+14.1^{\circ}$ (c $0.4, \mathrm{MeOH}$ ); ESI-MS $m / z$ : 463 $[\mathrm{M}+\mathrm{Na}]^{+}, 425,407,327,273,69$; the ${ }^{1} \mathrm{H}-\mathrm{NMR}$ and ${ }^{13} \mathrm{C}-\mathrm{NMR}$ data in accordance with the literature [23]. ${ }^{1} \mathrm{H}-\mathrm{NMR}: 1.45(1 \mathrm{H}, \mathrm{m}, 1 \alpha-\mathrm{H}), 1.86(1 \mathrm{H}, \mathrm{dd}, J=13.1,3.3 \mathrm{~Hz}, 1 \beta-\mathrm{H}), 1.75(1 \mathrm{H}, \mathrm{m}, 2 \alpha-\mathrm{H}), 1.67$ (1H, m, $2 \beta-\mathrm{H}), 3.29(1 \mathrm{H}, \mathrm{dd}, J=4.6,11.6 \mathrm{~Hz}, 3-\mathrm{H}), 1.67(1 \mathrm{H}, \mathrm{m}, 5-\mathrm{H}), 2.41(1 \mathrm{H}, \mathrm{dd}, J=3.9,15.8 \mathrm{~Hz}, 6 \alpha-\mathrm{H})$, $2.38(1 \mathrm{H}, \mathrm{dd}, J=12.4,15.8 \mathrm{~Hz}, 6 \beta-\mathrm{H}), 2.37(1 \mathrm{H}, \mathrm{m}, 11 \alpha-\mathrm{H}), 2.24(1 \mathrm{H}, \mathrm{m}, 11 \beta-\mathrm{H}), 1.76(1 \mathrm{H}, \mathrm{m}, 12 \alpha-\mathrm{H})$, $1.80(1 \mathrm{H}, \mathrm{m}, 12 \beta-\mathrm{H}), 1.56(1 \mathrm{H}, \mathrm{m}, 15 \alpha-\mathrm{H}), 2.13(1 \mathrm{H}, \mathrm{m}, 15 \beta-\mathrm{H}), 1.33(1 \mathrm{H}, \mathrm{m}, 16 \alpha-\mathrm{H}), 1.93(1 \mathrm{H}, \mathrm{m}$, $16 \beta-\mathrm{H}), 1.43(1 \mathrm{H}, \mathrm{m}, 17-\mathrm{H}), 0.72(3 \mathrm{H}, \mathrm{s}, 18-\mathrm{H}), 1.05(3 \mathrm{H}, \mathrm{s}, 19-\mathrm{H}), 1.43(1 \mathrm{H}, \mathrm{m}, 20-\mathrm{H}), 0.88(3 \mathrm{H}, \mathrm{d}$, $J=6.1 \mathrm{~Hz}, 21-\mathrm{H}), 1.13,1.56\left(2 \mathrm{H}, \mathrm{m}, 22-\mathrm{CH}_{2}\right), 1.90,2.04\left(2 \mathrm{H}, \mathrm{m}, 23-\mathrm{CH}_{2}\right), 5.58(1 \mathrm{H}, \mathrm{m}, 24-\mathrm{H}), 1.68$ $(3 \mathrm{H}, \mathrm{s}, 26-\mathrm{H}), 1.61(3 \mathrm{H}, \mathrm{s}, 27-\mathrm{H}), 0.99(3 \mathrm{H}, \mathrm{s}, 28-\mathrm{H}), 0.88(3 \mathrm{H}, \mathrm{s}, 29-\mathrm{H}), 0.97(3 \mathrm{H}, \mathrm{s}, 30-\mathrm{H}) .{ }^{13} \mathrm{C}-\mathrm{NMR}$ : 34.6 (C-1), 27.4 (C-2), 78.0 (C-3), 38.8 (C-4), 48.2 (C-5), 35.8 (C-6), 198.3 (C-7), 138.9 (C-8), 165.4 (C-9), 39.3 (C-10), 23.7 (C-11), 29.9 (C-12), 44.6 (C-13), 47.7 (C-14), 31.4 (C-15), 28.7 (C-16), 48.2 (C-17), 15.7 (C-18), 18.6 (C-19), 35.6 (C-20), 18.8 (C-21), 35.5 (C-22), 24.7 (C-23), 125.1 (C-24), 139.4 (C-25), 25.7 (C-26), 17.7 (C-27), 27.3 (C-28), 15.1 (C-29), 24.4 (C-30).

(24R)-Eupha-8,25-diene-3 $\beta, 24$-diol (5), Formula: $\mathrm{C}_{30} \mathrm{H}_{50} \mathrm{O}_{2}$; colorless oil; $[\alpha]_{\mathrm{D}}{ }^{20}+5.1^{\circ}$ (c $\left.0.30, \mathrm{MeOH}\right)$; ESI-MS $m / z$ : $465[\mathrm{M}+\mathrm{Na}]^{+}$; the ${ }^{1} \mathrm{H}-\mathrm{NMR}$ and ${ }^{13} \mathrm{C}-\mathrm{NMR}$ data in accordance with the literature [24]. ${ }^{1} \mathrm{H}-\mathrm{NMR}: 4.92,4.83\left(2 \mathrm{H}\right.$, br s, 26- $\left.\mathrm{CH}_{2}\right), 4.01(1 \mathrm{H}, \mathrm{t}, J=6.1 \mathrm{~Hz}, 24-\mathrm{H}), 3.23(1 \mathrm{H}, \mathrm{dd}, J=4.5,11.7 \mathrm{~Hz}$, 3-H), 1.72 (3H, br s, 27-H), 0.99, 0.94, 0.86 (each 3H, s, $\left.\mathrm{CH}_{3}\right), 0.85$ (3H, d, J=6.2 Hz, 21-H), 0.79, 0.75 (each 3H, s, $\left.\mathrm{CH}_{3}\right)$; ${ }^{13} \mathrm{C}-\mathrm{NMR}: 35.3$ (C-1), 27.9 (C-2), 79.0 (C-3), 38.9 (C-4), 51.0 (C-5), 18.9 (C-6), 27.7 (C-7), 134.0 (C-8), 133.5 (C-9), 37.3 (C-10), 21.5 (C-11), 30.9 (C-12), 44.1 (C-13), 50.0 (C-14), 31.1 (C-15), 28.0 (C-16), 49.7 (C-17), 15.7 (C-18), 20.1 (C-19), 36.0 (C-20), 19.0 (C-21), 37.3 (C-22), 31.6 (C-23), 76.6 (C-24), 147.6 (C-25), 111.1 (C-26), 17.4 (C-27), 28.0 (C-28), 15.5 (C-29), 24.5 (C-30).

(20R,23E)-Eupha-8,23-diene-3 $\beta, 25$-diol (6), Formula: $\mathrm{C}_{30} \mathrm{H}_{50} \mathrm{O}_{2}$; colorless needles; m.p. $132-133{ }^{\circ} \mathrm{C}$; $[\alpha]_{\mathrm{D}}{ }^{20}+17.0^{\circ}$ (c $0.45, \mathrm{CHCl}_{3}$ ); ESI-MS $m / z: 465[\mathrm{M}+\mathrm{Na}]^{+}$; the ${ }^{1} \mathrm{H}-\mathrm{NMR}$ and ${ }^{13} \mathrm{C}-\mathrm{NMR}$ data in accordance with the literature [24]. ${ }^{1} \mathrm{H}-\mathrm{NMR}: 5.58(2 \mathrm{H}$, br s, $\mathrm{H}-23$ and $\mathrm{H}-24), 3.23(1 \mathrm{H}, \mathrm{dd}, J=4.5$, $11.7 \mathrm{~Hz}, 3 \alpha-\mathrm{H}), 2.34(1 \mathrm{H}$, br d, $J=12.7 \mathrm{~Hz}, 5-\mathrm{H}), 1.31(6 \mathrm{H}, \mathrm{s}, 26-\mathrm{H}$ and 27-H), 1.00, 0.95, 0.88 (each $3 \mathrm{H}, \mathrm{s}, 30-\mathrm{H}, 28-\mathrm{H}$, and 29-H), $0.82(3 \mathrm{H}, \mathrm{d}, J=6.0 \mathrm{~Hz}, 21-\mathrm{H}), 0.80,0.78$ (each $3 \mathrm{H}, \mathrm{s}, 19-\mathrm{H}$ and 18-H). ${ }^{13}$ C-NMR: 35.3 (C-1), 27.9 (C-2), 79.0 (C-3), 38.9 (C-4), 51.0 (C-5), 18.9 (C-6), 27.7 (C-7), 134.0 (C-8), 133.5 (C-9), 37.3 (C-10), 21.5 (C-11), 31.0 (C-12), 44.2 (C-13), 50.0 (C-14), 29.8 (C-15), 27.9 (C-16), 49.6 (C-17), 15.8 (C-18), 20.2 (C-19), 35.7 (C-20), 19.1 (C-21), 37.3 (C-22), 125.7 (C-23), 139.3 (C-24), 70.8 (C-25), 29.9 (C-26), 29.9 (C-27), 28.1 (C-28), 15.5 (C-29), 24.5 (C-30). 


\subsection{Computational Methods}

AutoDock Vina 1.1.2 [25] was used for all dockings in this study. The $1.80 \AA \mathrm{X}$-ray structure human 11 $\beta$-HSD1 complexed with inhibitor (PDB code: 3TFQ) was chosen as receptor in docking simulations. The Ligand and receptor were prepared with MGLTools 1.5.2. In general, the docking parameters for Vina were kept to their default values. The center of docking-grid is located on the centric of the corresponding ligand. The top 10 poses of each compound were reserved for the binding mode analysis. DiscoveryStudio 2.5.5 was used to build up the sequence alignment between human $11 \beta$-HSD1 and HSD2. The final structures were analyzed using PyMOL [26].

\subsection{Biological Testing of Compounds}

Glucocorticoid hormones play important roles in many biological and physiological processes, including regulation of energy metabolism; inflammatory, immune and stress responses; and cardiovascular homeostasis. The action of glucocorticoid on target tissue is not inevitable dependent on the circulating levels, but is regulated in a tissue-specific manner by the $11 \beta$-hydroxysteroid

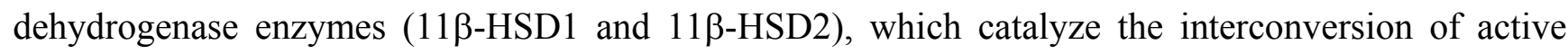
11-hydroxyglucocorticoids (cortisol in human and corticosterone in rodent) and their respective inert 11-keto forms (cortisone in human and 11-dehyfrocorticosterone in rodent) [27]. 11 $\beta$-HSD1 is highly expressed in liver, gonad, adipose tissue and brain, where it acts as a reductase regenerating the active glucocorticoids from its inactive forms, thus amplifies local glucocorticoid action [28]. 11ß-HSD2 is predominantly expressed in aldosterone target cells such as kidney and colon, where it catalyses the inactivation of glucocorticoids, thereby preventing excessive activation of the mineralocorticoid receptor and sequelae including sodium retention, hypokalemia, and hypertension. We tested the

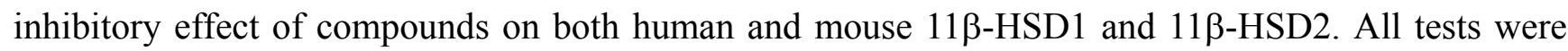

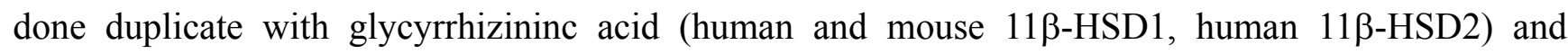
carbenoxolone (mouse $11 \beta$-HSD2) as positive control. $\mathrm{IC}_{50}(\mathrm{X} \pm \mathrm{S} . \mathrm{D} ., \mathrm{n}=2$ ) values were calculated by using Prism Version 4 (GraphPad Software, San Diego, CA, USA.).

Inhibition of compounds on human or mouse $11 \beta$-HSD1 and 11 1 -HSD2 enzymatic activities were determined by the scintillation proxitimity assay (SPA) using microsomes containing 11 $\beta$-HSD1 or $11 \beta$-HSD2 according to the previous studies [29]. Briefly the full-length cDNAs of human or marine $11 \beta$-HSD1 and $11 \beta$-HSD2 were isolated from the cDNA libraries provided by NIH Mammalian Gene Collection and cloned into pcDNA3 expression vector. HEK-293 cells were transfected with the pcDNA3-dericed expression plasmid and selected by cultivation in the presence of $700 \mu \mathrm{g} / \mathrm{mL}$ of G418. The microsomal fraction overexpressing $11 \beta$-HSD1 or $11 \beta-H S D 2$ was prepared from the HEK-293 cells stable transfected with either $11 \beta$-HSD1 or $11 \beta$-HSD2 and used as the enzyme source for SPA. Microsomes containing human or mouse $11 \beta$-HSD1 was incubated with NADPH and $\left[{ }^{3} \mathrm{H}\right]$ cortisone, then the product, $\left[{ }^{3} \mathrm{H}\right]$ cortisol was specifically captured by a monoclonal antibody coupled to protein A-coated SPA beads. The $11 \beta$-HSD2 screening was performed by incubating $11 \beta$-HSD2 microsomes with $\left[{ }^{3} \mathrm{H}\right]$ cortisol and $\mathrm{NAD}^{+}$and monitoring substrate disappearance. $\mathrm{IC}_{50}(\mathrm{X} \pm \mathrm{S} . \mathrm{D} ., \mathrm{n}=2)$ values were calculated by using Prism Version 4 (GraphPad Software, San Diego, CA, USA.) with glycyrrhizininc acid and carbenoxolone as positive control. 


\section{Conclusions}

We have described the isolation and characterization of six euphane-type triterpenoids from the plant Euphorbia kansui. All compounds were fully characterized using $\mathrm{MS},{ }^{1} \mathrm{H}-$ and ${ }^{13} \mathrm{C}-\mathrm{NMR}$ spectroscopic techniques. In bioassay studies, all the compounds exhibited strong inhibitory activity

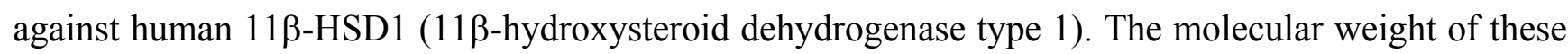
six compounds is less than 500 and the number of hydrogen bond donors and acceptors is less than 5 . In conclusion, the obtained results indicate that E. kansui exhibits potent inhibitory activities against human $11 \beta$-HSD1 which might be useful for therapeutic purposes to prevent type 2 diabetes, and could be used as a potential antidiabetic agent for treatment of diabetes.

\section{Supplementary Materials}

Supplementary materials can be accessed at: http:/www.mdpi.com/1420-3049/17/10/11826/s1.

\section{Acknowledgments}

We thank Xun Gong of the Kunming Institute of Botany, Chinese Academy of Sciences, for identification of the plant material.

\section{Conflict of Interest}

The authors declare no conflict of interest.

\section{References}

1. Stumvoll, M.; Goldstein, B.J.; van Haeften, T.W. Type 2 diabetes: Principles of pathogenesis and therapy. Lancet 2005, 365, 1333-1346.

2. Yang, W.Y.; Lu, J.M.; Weng, J.P.; Jia, W.P.; Ji, L.N.; Xiao, J.Z.; Shan, Z.Y.; Liu, J.; Tian, H.M.; Ji, Q.H.; et al. Prevalence of Diabetes among Men and Women in China. N. Engl. J. Med. 2010, 12, 1090-1101.

3. Evans, F.J.; Taylor, S.E. Pro-inflammatory, Tumour-promoting and anti-tumour diterpenes of the plant families Euphorbiaceae and Thymelaeaceae. Prog. Chem. Org. Nat. Prod. 1983, 44, 1-99.

4. Günther, G.; Martinek, T.; Dombi, G.; Hohmann, J.; Vasas, A. Structural characterisation and dynamic NMR studies of a new peracylated macrocyclic diterpene. Magn. Reson. Chem. 1999, 37, 365-370.

5. Appendino, G.; Belloro, E.; Tron, G.C.; Jakupovic, J.; Ballero, M. Diterpenoids from Euphorbia pithyusa subsp. cupanii. J. Nat. Prod. 1999, 10, 1399-1404.

6. Vogg, G.; Mattes, E.; Rothenburger, J.; Hertkorn, N.; Achatz, S.; Sanderman, H. Tumor promoting diterpenes from Euphorbia leuconeura L. Phytochemistry 1999, 2, 289-295.

7. He, W.D.; Cik, M.; Lesage, A.; Linden, I.V.D.; Kinpe, N.D.; Appendino, G.; Bracke, J.; Mathenge, S.G.; Mudida, F.P.; Leysen, J.E.; et al. Kirkinine, A New Daphnane Orthoester with Potent Neurotrophic Activity from Synaptolepis kirkii. J. Nat. Prod. 2000, 9, 1185-1187. 
8. Amit, R.; Shailendra, S. Limonoids: Overview of Significant Bioactive Triterpenes Distributed in Plants Kingdom. Biol. Pharm. Bull. 2006, 2, 191-201.

9. Guo, J.; Fang, X.; Di, Y.T.; Hua, H.M.; Hao, X.J. Kansuinine J, A new macrocyclic diterpenoid from the roots of Euphorbia kansui. Chin. Chem. Lett. 2010, 8, 943-946.

10. Halaweish, F.T.; Kronberg, S.; Rice, J.A. Rodent and Ruminant Ingestive Response to Flavonoids in Euphorbia esula. J. Chem. Ecol. 2002, 5, 1073-1082.

11. Liu, L.G.; Meng, J.C.; Tan, R.X. New macrocyclic diterpenoids from Euphorbia esula. Planta Med. 2002, 3, 244-248.

12. Akihisa, T.; Kithsiri Wijeratne, E.M.; Tokuda, H.; Enjo, F.; Toriumi, M.; Kimura, Y.; Koike, K.; Nikaido, T.; Tezuka, Y.; Nishino, H. Eupha-7,9(11),24-trien-3 $\alpha$-ol (“Antiquol C”) and Other Triterpenes from Euphorbia antiquorum Latex and Their Inhibitory Effects on Epstein-Barr Virus Activation. J. Nat. Prod. 2002, 65, 158-162.

13. Jiang, Z.H.; Tanaka, T.; Hirata, H.; Fukuoka, R.; Kouno, I. The first euphane-type triterpene tridesmosides and bisdesmoside from Rhoiptelea chiliantha. Tetrahedron 1997, 50, 16999-17008.

14. Akihisa, T.; Kimura, Y.; Kokke, W.C.M.C.; Takase, S.; Yasukawa, K.; Tamura, T. Tirucalla-

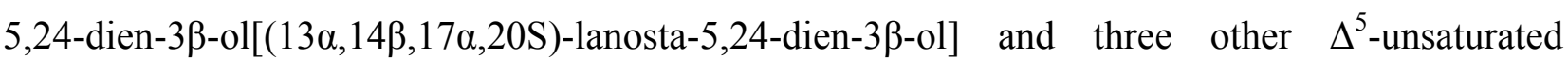
tirucallanes from the roots of Bryonia dioica Jacq.: The first naturally occurring C-10 methylated tetracyclic triterpene alcohols with a $\Delta^{5}$-monounsaturated skeleton. J. Chem. Soc. Perk. Trans. 1996, 19, 2379-2384.

15. Arai, Y.; Hirohara, M.; Ageta, H. Fern constituents: Three new skeletal triterpenoid hydrocarbons isolated from Polypodiodes niponica. Tetrahedron Lett. 1989, 30, 7209-7212.

16. Abe, I.; Rohmer, M. Enzymic cyclization of 2,3-dihydrosqualene and squalene 2,3-epoxide by squalene cyclases: From pentacyclic to tetracyclic triterpenes. J. Chem. Soc. Perk. Trans. 1994, 7 , 783-791.

17. Mamta, M.; Yogendra, N.; Sushil, K. Euphane triterpenoid and lipid constituents from Butea monosperma. Phytochemistry 2000, 54, 835-838.

18. Ge, R.; Huang, Y.; Liang, G.; Li, X. 11ß-hydroxysteroids dehydrogenase type 1 inhibitors as promising therapeutic drugs for diabetes: Status and development. Curr. Med. Chem. 2010, 5, $412-422$.

19. Chen, Q.; Li, Y.D.; Li, K.L. Clinical study on the effect of Tangwei Dressing applied to acupoint on gastrointestinal hormones of patients with diabetic gastric paresis. Chin. Tradit. Patent Med. 2011, 33, 208-211.

20. Gao, J.M.; Bosco, D.A.; Powers, E.T.; Kelly, J.W. Localized thermodynamic coupling between hydrogen bonding and microenvironment polarity substantially stabilizes proteins. Nat. Struct. Mol. Biol. 2009, 16, 684-690.

21. Guo, J.; He, H.P.; Fang, X.; Di, Y.T.; Li, S.L.; Zhang, Z.; Leng, Y.; Hua, H.M.; Hao, X.J. Kansuinone, A novel triterpene with an unusual skeleton from Euphorbia kansui. Tetrahedron Lett. 2010, 48, 6286-6289.

22. Cao, D.; Su, Y.L.; Yang, J.S. Triterpene Constitutents From Euphorbia nematocypha HAND-MAZZ. Acta Pharm. Sin. 1992, 6, 445-451. 
23. Wang, L.Y.; Wang, N.L.; Yao, X.S.; Syohei, M.; Susumu, K. Euphane and Tirucallane Triterpenes from the Roots of Euphorbia kansui and Their in Vitro Effects on the Cell Division of Xenopus. J. Nat. Prod. 2003, 66, 630-633.

24. Leong, Y.W.; Harrison, L.J. (20R, 23E)-Eupha-8,23-diene-3,25-diol from Tripetalum cymosum. Phytochemistry 1999, 5, 849-857.

25. Ttott, O.; Olson, A.J. AutoDock Vina: Improving the speed and accuracy of docking with a new scoring function, efficient optimization and multithreading. J. Comp. Chem. 2010, 31, 455-461.

26. DeLano, W.L. The PyMOL Molecular Graphics System. Available online: http://www.pymol.org (accessed on 14 August 2009).

27. Stewart, P.M.; Draper, N. 11ß-Hydroxysteroid dehydrogenase and the pre-receptor regulation of corticosteroid hormone action. J. Endocrinol. 2005, 186, 251-271.

28. Seckl, J.R.; Walker, B.R. Minireview: 11ß-hydroxysteroid dehydrogenase type 1-A tissue-specific amplifier of glucocorticoid action. Endocrinology 2001, 142, 1371-1376.

29. Yang, H.Y.; Dou, W.; Lou, J.; Leng, Y.; Shen, J.H. Discovery of novel inhibitors of 11beta-hydroxysteroid dehydrogenase type 1 by docking and pharmacophore modeling. Bioorg. Med. Chem. Lett. 2008, 18, 1340-1345.

Sample Availability: Contact the authors.

(C) 2012 by the authors; licensee MDPI, Basel, Switzerland. This article is an open access article distributed under the terms and conditions of the Creative Commons Attribution license (http://creativecommons.org/licenses/by/3.0/). 